\section{Putting the protists in their place}

\author{
F.E.G. Cox
}

An Illustrated Guide to the Protozoa. Edited by John J. Lee, Seymour H. Hutner and Eugene C. Bovee. Society of Protozoologists, PO Box 368, Lawrence, Kansas 66044, USA: 1985. Pp.629. $\$ 80$ (non-members of the Society), $\$ 60$ (members).

ONLY after a long gestation period has this mammoth publication finally appeared. It comes in large format, printed on glossy paper, and is illustrated with over 3,000 line drawings, photographs, and transmission and scanning electron micrographs. The stated objective was to produce a book "which would enable scientists and students working with protists to place them in an up to date taxonomic scheme". Certainly, every protozoologist should have a copy (or access to one) but whether it will be used as a continual source of reference is debatable.

In general, the 1980 Society of Protozoologists classification (the Levine scheme) has been used, with minor modifications to the phyla Sarcomastigophora, Labyrinthomorpha, Apicomplexa and Myxozoa, major changes to the Microspora and a completely new classification of the Ciliophora. In other respects, the 23 authors have adopted different approaches. For some groups, particularly among the Phytomastigophorea, the reader is taken to species, in others, for example the Ciliophora, the genus, and for some, including the Foraminifera, the family. For the Ciliophora, there is an overall dichotomous key that leads to the order followed by subsequent keys to family. Other groups have keys starting with higher taxa and some, notably the Apicomplexa, have none.

Who is this book for? Certainly not the novice. I had difficulty in following some of the keys and had to resort to the welltried technique of finding a suitable picture and working backwards. The editors point out that the original literature will have to be consulted, and most protozoologists concerned with taxonomic problems will do this automatically. But those who are not experts, or do not have access to library facilities, might be tempted to use the book as a substitute for the original literature.

Parasitologists, too, may find it a bit disappointing. Those interested in trypanosomes will be alarmed to read on p.9 that Trypanosoma gambiense is a parasite of rodents, and on p.142 that the flagellum does not arise from a flagellar pocket (thus distorting the whole of the key), and will be dismayed by the diagram of "Trypano- soma congolense" (p.151) which is actually Cryptobia vaginalis. Unfortunately, this cannot be checked because the reference given is not actually cited in full and is in fact wrong. Other such errors limit the book's value considerably.

Overall, this guide represents a heroic effort which does not quite come off; in retrospect, a series of specialist keys, which could be revised easily, might have been better. Treated with caution, however, this is a nice book to have around and we shall never see its like again.

F.E.G. Cox is a Professor in theDepartment of Biophysics, Cell and Molecular Biology, King's College London $(K Q C)$, London WC2R $2 L S$, UK.

\section{Threads of insight}

\section{John J. O'Reilly}

Fiber Optics: Technology and Applications. By Stewart D. Personick. Plenum:1985. Pp.257. \$45, £38.03.

STEWART Personick has been a leading figure in optical fibre research for some 15 years, and his early papers on receiver design for digital fibre optic systems are still the key references in this area. A new work by such an eminent author is thus well deserving of special attention. The book is an outgrowth of an introductory course on the subject and, consequently, the author does not go into much detail. Rather, he presents first, in Part I, an overview of components and subsystems with a view to providing a basis upon which the reader can build an understanding of the practical applications and limitations surveyed in Part II.

In addition to the usual material on principles, sources and detectors, Part I includes helpful discussions of practical transmitter drive circuits, receiver design and optical components. System impairments arising from interactions between the source and the fibre, such as modal noise, mode partition, modal distortion and laser noise induced by reflections, are also outlined.

The applications considered in Part II range widely, encompassing telecommunications trunk transmission systems, data links, local area networks, and analogue and broadband networks. Sensing systems are covered briefly, as are system measurements. There is, too, a very brief account of emerging technologies such as integrated optoelectronics, coherent systems and optical switching.

The book is well provided with diagrams and photographs which should enhance its usefulness for the novice. In some instances, though, the reproduction process has resulted in an unfortunate lack of detail (and on one or two occasions the author refers to the colours in monochrome photographs!). The text is largely non-mathematical in nature and there is little in the way of fundamental analysis. For all that, this is not a lightweight book; what it lacks in rigour it makes up for in practical insight and experience.

The descriptions are clear, concise and simple, enabling the underlying principles to be readily appreciated, while references are provided to enable the reader to follow up on the details. The book does not, however, offer us anything new; indeed, several sections of the early chapters seem to have much in common with the author's previous book, Optical Fiber Transmission Systems, which was published in 1981 as a volume in the same series.

All in all, this is an interesting collection of topics, pitched at a level which will allow a newcomer to the subject to get to grips with the basic ideas and gain some insight into industrial practice. But, for me, it lacks the style and flair which characterize so much of Dr Personick's output in the technical journals.

John J. O'Reilly is Professor in the School of Electronic Engineering Science, University College of North Wales, Bangor, Gwynedd LL57 $1 U T, U K$.

Fechner's colours: as the eye scans across the diagram, the spatial pattern is translated into a temporal pattern of stimulation. Because the different cones and chromatic channels respond at different rates, unsaturated colours may be seen. The illustration is taken from a new textbook published by Harper \& Row, Seeing the Light: Optics in Nature, Photography, Color, Vision, and Holography, by David Falk, Dieter Brill and David Stork. Price is \$35.50, $\$ 31.95$. 